\title{
How to Predict the Impact of Methylphenidate on Cardiovascular Risk in Children with Attention Deficit Disorder: Methylphenidate Improves Autonomic Dysfunction in Children with ADHD
}

\author{
Reiner Buchhorn, ${ }^{1}$ Christian Müller, ${ }^{1}$ Christian Willaschek, ${ }^{1}$ and Kambiz Norozi ${ }^{2,3}$ \\ ${ }^{1}$ Department of Paediatrics, Caritas Hospital, 97980 Bad Mergentheim, Germany \\ ${ }^{2}$ Department of Paediatrics, The University of Western Ontario, London, ON, Canada N6A 5W9 \\ ${ }^{3}$ Department of Pediatric Cardiology, Hannover Medical School, 30625 Hannover, Germany
}

Correspondence should be addressed to Reiner Buchhorn, reiner.buchhorn@ckbm.de

Received 9 November 2011; Accepted 8 December 2011

Academic Editor: T. B. Vree

Copyright (C) 2012 Reiner Buchhorn et al. This is an open access article distributed under the Creative Commons Attribution License, which permits unrestricted use, distribution, and reproduction in any medium, provided the original work is properly cited.

Background. Although stimulants have long been touted as treatments for attention deficit disorder with or without hyperactivity (ADHD), in recent years, increasing concerns have been raised about the cardiovascular safety of these medications. We aimed to prove if measurements of autonomic function with time domain analysis of heart rate variability (HRV) in 24-hour Holter ECG are useful to predict the risk of sudden cardiac death in ADHD children and adolescents. Methods. We analysed HRV obtained from children with the diagnosis of ADHD prior to $(N=12)$ or during medical therapy $(N=19)$ with methylphenidate $(\mathrm{MPH})$, aged $10.8 \pm 2.0$ years (mean \pm SD), who were referred to our outpatient Paediatric Cardiology Clinic to rule out heart defect. As a control group, we compared the HRV data of 19 age-matched healthy children without heart defect. Results. Average HRV parameters from 24-hour ECG in the ADHD children prior to MPH showed significant lower values compared to healthy children with respect to rMSSD $(26 \pm 4 \mathrm{~ms}$ versus $44 \pm 10 \mathrm{~ms}, P \leq 0.0001)$ and pNN50 $(6.5 \pm 2.7 \%$ versus $21.5 \pm 9.0 \%, P \leq 0.0001)$. These values improved in MPH-treated children with ADHD (RMSSD: $36 \pm 8$ ms; pNN50: $14.2 \pm 6.9 \%$ ). Conclusion. Children who suffer from ADHD show significant changes in HRV that predominantly reflects diminished vagal tone, a well-known risk factor of sudden cardiac death in adults. In our pilot study, MPH treatment improved HRV.

\section{Introduction}

Reports of sudden death among children and adolescents receiving stimulant medications for treatment of attention deficit disorder with or without hyperactivity (ADHD) have raised concerns about the safety of these agents. In the last two years, many articles have been published about the cardiovascular safety of stimulant medication [16]. There are very few reports about clinically measured cardiovascular data in ADHD children and adolescents $[7,8]$. It remains controversial if treatment of ADHD children with stimulants reduces or increases the risk of sudden death (SD) [6]. The assumption that stimulant-treated ADHD patients are at higher risk for SD is based on either (a) the proarrhythmogenic effects of these agents, which are closely related members of the class of sympathomimetic amines, or (b) complicated statistical models with estimated data from uncertain data bases like the Adverse Event Reporting System. Due to the rarity of SD in pediatric populations, compared to adults, it is extremely difficult to establish a reliable model to predict sudden cardiac arrest in children and adolescents. It is the search for the needle in the haystack [2]. For the assessment of cardiac risk, there is no doubt that an accurate history and physical examination are important, but ambiguities in current screening recommendations with respect to electrocardiogram assessment show the lack of sufficient surrogate measurements [9].

The autonomic nervous system plays an important role in sudden cardiac death [10]. Twenty-four-hour Holter recordings in post myocardial infarction (MI) in adult 
patients showed that depressed heart rate variability (HRV) was a significant predictor of mortality after adjusting for clinical and demographic features, including ejection fraction. These studies were further confirmed by others studying post-MI patients, who showed that impaired HRV was an independent predictor of cardiac mortality only within 6 months of MI and seemed to improve over time $[10,11]$. HRV is also reduced in survivors of sudden cardiac death not associated with coronary artery disease and may be related to the decrease of parasympathetic tone [12].

Measurement of heart rate variability in 24-hour Holter ECG is completely noninvasive. Normal values are available for children [13] and adults [14].

In our pilot study, we investigate whether measurements of autonomic function with time domain analysis of HRV in 24-hour ECG are useful to determine risk factors for sudden cardiac death in patients with ADHD.

\section{Methods}

2.1. Subjects. We analysed HRV obtained from children with the diagnosis of ADHD prior to $(N=12)$ or during medical therapy $(N=19)$ with methylphenidate (MPH), aged $10.8 \pm 2.0$ years. From August 2005 to Mach 2010, children with the diagnosis of ADHD were referred to our Paediatric Cardiology Outpatient Clinic for cardiology assessment. The mean daily MPH dosage of 19 children on stimulant medication was $0.8 \pm 0.4 \mathrm{mg} / \mathrm{kg} /$ day ( 9 children received methylphenidate extended release). All children received a standard 12-lead ECG, an echocardiogram, and 24-hour Holter ECG. As a control group, we compared the HRV data of 19 age-matched healthy children, who were referred to rule out cardiac defects due to heart murmur, palpitation, or chest pain.

\subsection{Processing and Analysis of 24-Hour Holter Recordings.} Autonomic control of cardiovagal function was assessed by time domain analysis of 24-hour ambulatory digital recordings of the electrocardiogram using a two-channel Holter monitor (Pathfinder, Spacelabs). All Holter recordings were reviewed by the same cardiologist and were edited to validate the system's QRS labelling. Measures of HRV were calculated employing only normal to normal intervals. Numbers of pairs of adjacent normal beat (NN) intervals differing by more than $50 \mathrm{~ms}$ were given as the percentage of the total number of all NN intervals during 24 hours (pNN50).

Measurement and physiological interpretation of HRV parameters were performed according to the standards of the Task Force of the European Society of Cardiology and the North American Society of Pacing and Electrophysiology [15]. RMSSD, pNN50, and heart rate reflect predominantly a response to changes in vagal tone. SDNN is dually influenced by cholinergic and adrenergic activity, as well as other physiological inputs.

2.3. Exclusion Criteria. The data of 5 children with ADHD and more than 20 premature ventricular contractions per hour were excluded.
2.4. Informed Consent. After adequate explanation of the purpose of the study, informed consent was obtained from the parents. The study protocol was approved by the local ethic committee before the start of the investigation.

2.5. Statistical Analysis. All results are reported as mean \pm standard deviation. Because all variables were normally distributed, a parametric test was used. Differences between the controls and ADHD patients with and without $\mathrm{MPH}$ treatment were tested with an unpaired $t$-test with Welch's correction. For all parameters, a value of $P<0.05$ was considered statistically significant. The data analyses were performed using Excel 2003 (Microsoft, USA) and Prism (GraphPad software Inc., USA).

\section{Results}

All patients were in sinus rhythm and had a normal echocardiogram. As shown in Table 1, there is no significant statistical difference with respect to age between the ADHD patients (with or without MPH) and the healthy control group. Average HRV parameters from the 24-hour ECG in the ADHD children without MPH show significantly lower heart rate variability compared to the healthy children with respect to $\mathrm{rMSSD}(26 \pm 4 \mathrm{~ms}$ versus $44 \pm 10 \mathrm{~ms}, P \leq 0.0001)$ and pNN50 $(6.5 \pm 2.7 \%$ versus $21.5 \pm 9.0 \%, P \leq 0.0001)$. These parameters improve significantly after MPH treatment (rMSSD: $36 \pm 8$ ms; pNN50: 14.2 $\pm 6.9 \%$ ). These parameters predominantly reflect changes in vagal tone. Due to these autonomic changes, untreated children with ADHD had a higher mean heart rate compared to healthy children (94 \pm $7 / \mathrm{min}$ versus $85 \pm 10 / \mathrm{min})$. Average SDNN in ADHD patients was not significantly different from healthy children.

\section{Discussion}

In our pilot study, we measured time domain values of heart rate variability in 24-hour Holter ECGs in children with ADHD. Our data demonstrate significantly lower rMSSD and pNN50 values and elevated heart rates in nonpharmacologically treated ADHD children and adolescents. These parameters predominantly reflect diminished parasympathic activity. If we adjust the data of our 12 untreated children with ADHD in the figure of published rMSSD and pNN50 normal values in children [13] and adults [14] (Figure 1), we notice that these data are not only far away from the mean values for healthy children between 8 and 13 years but also near to the dashed line, which represents cutoff for increased risk of sudden cardiac death in adults. Furthermore, we demonstrate significant improvement and almost normal values of rMSSD and pNN50 after treatment with MPH.

Our data indicate the lack of physiological maturation of autonomic function with low parasympathetic tone in children with $\mathrm{ADHD}$ at the average age of 10 years. From a pathophysiological point of view, patients with low parasympathic activity have an increased risk for sudden cardiac death, independent of other risk factors. This might explain the increased baseline risk in these patients observed 
TABLE 1: Heart rate variability in healthy children $(n=19)$, children with ADHD untreated $(n=12)$ and treated with methylphenidate $(\mathrm{MPH})(n=19)$

\begin{tabular}{|c|c|c|c|c|}
\hline & Mean \pm SD & $P 1$ versus 2 & $P 2$ versus 3 & $P 1$ versus 3 \\
\hline \multicolumn{5}{|l|}{ Age (years) } \\
\hline Healthy & $10.8 \pm 3.5$ & & & \\
\hline ADHD & $10.8 \pm 2.0$ & $P=\mathrm{ns}$ & $P=\mathrm{ns}$ & $P=\mathrm{ns}$ \\
\hline $\mathrm{ADHD}+\mathrm{MPH}$ & $10.6 \pm 2.8$ & & & \\
\hline \multicolumn{5}{|l|}{ Heart rate (/min.) } \\
\hline Healthy & $85 \pm 10$ & & & \\
\hline ADHD & $94 \pm 7$ & $P=0.003$ & $P=0.04$ & $P=\mathrm{ns}$ \\
\hline $\mathrm{ADHD}+\mathrm{MPH}$ & $90 \pm 6$ & & & \\
\hline \multicolumn{5}{|l|}{ SDNN (ms) } \\
\hline Healthy & $146 \pm 30$ & & & \\
\hline ADHD & $136 \pm 41$ & $P=\mathrm{ns}$ & $P=\mathrm{ns}$ & $P=\mathrm{ns}$ \\
\hline $\mathrm{ADHD}+\mathrm{MPH}$ & $151 \pm 25$ & & & \\
\hline \multicolumn{5}{|l|}{$r M S S D(m s)$} \\
\hline Healthy & $44 \pm 10$ & & & \\
\hline ADHD & $26 \pm 4$ & $P<0.0001$ & $P=0.0001$ & $P=0.008$ \\
\hline $\mathrm{ADHD}+\mathrm{MPH}$ & $36 \pm 8$ & & & \\
\hline \multicolumn{5}{|l|}{ pNN50 (\%) } \\
\hline Healthy & $21.5 \pm 9.0$ & & & \\
\hline ADHD & $6.5 \pm 2.7$ & $P=0.0001$ & $P=0.0002$ & $P=0.008$ \\
\hline $\mathrm{ADHD}+\mathrm{MPH}$ & $14.2 \pm 6.9$ & & & \\
\hline
\end{tabular}

Values are given in mean \pm SD. Probability of difference using student $t$-test; significant values are in bold; SDNN: standard deviation of all NN intervals; rMSSD: square root of the mean of the sum of the squares of differences between adjacent NN intervals; pNN50: number of pairs of adjacent NN intervals differing by more than $50 \mathrm{~ms}$ divided by the total number of all NN intervals.

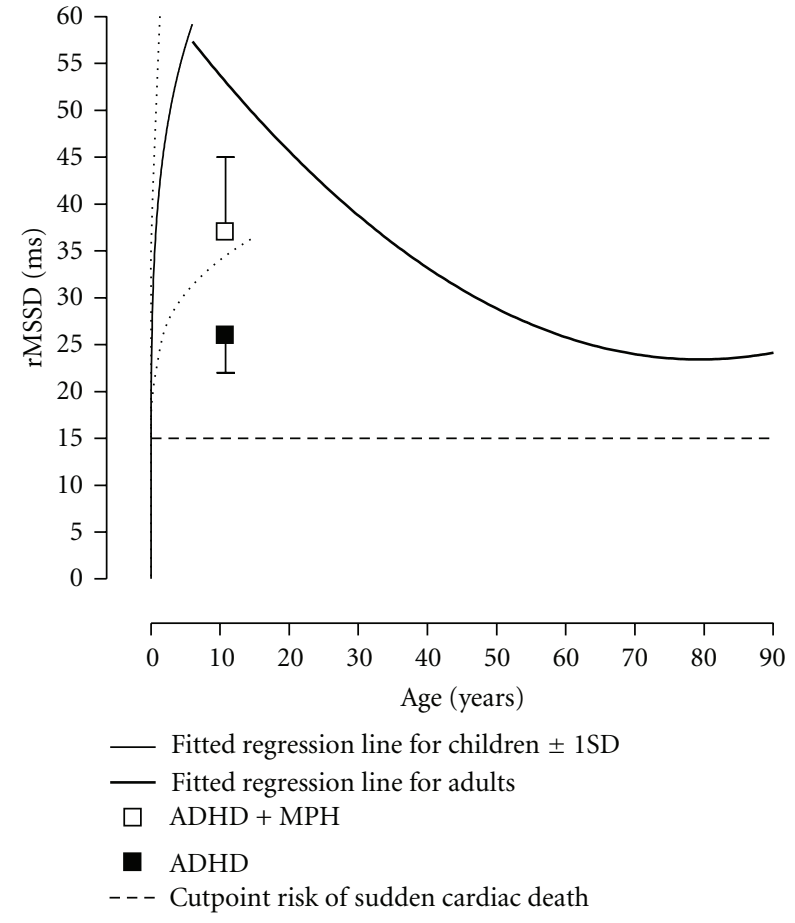

(a)

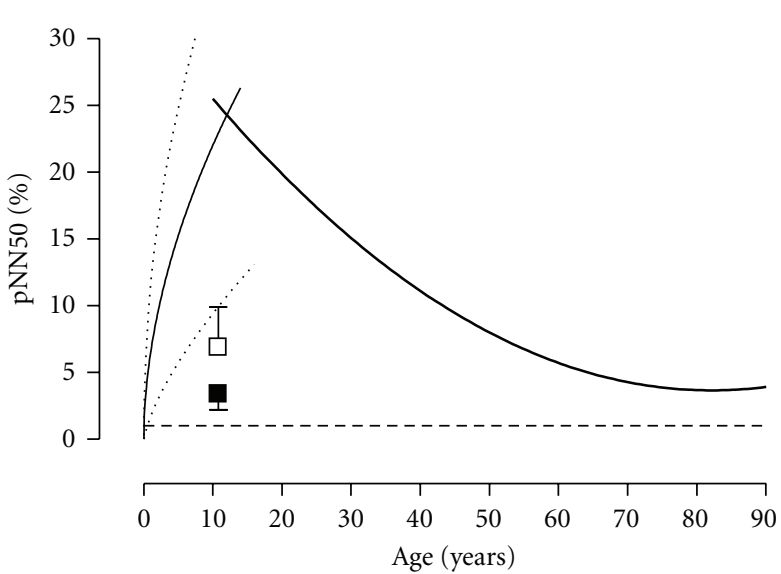

- Fitted regression line for children $\pm 1 \mathrm{SD}$

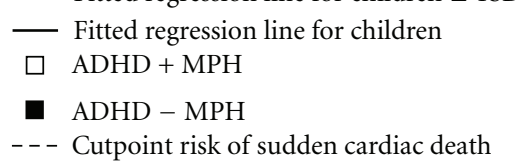

(b)

FIGURE 1: Relationship between age and the HRV parameter rMSSD and pNN50 in healthy subjects, published by Massin et al. [13] and Umetani et al. [14]. These parameters reflect parasympathetic activity of the autonomic nervous system. Solid lines: fitted regression lines \pm 1 SD line in childhood. Dashed line: published cutoffs for increased risk of mortality from sudden cardiac death. $\mathbf{\square}$ : Values of 12 children with ADHD and $\square: 19$ children with ADHD treated with methylphenidate. 
in former studies [3]. However, higher rMSSD and pNN50 values in our children with ADHD who were treated with $\mathrm{MPH}$ indicate a higher vagal tone, which is considered as protective for sudden cardiac death [10]. Our data supply the first feasible explanation why recently published populationbased cohort studies showed an apparent protective association and not an increased risk of serious cardiovascular events in stimulant users [16-18]. These results need to be proven by randomized prospective studies.

In conclusion, our data provides new information about autonomic dysfunction in children with $\mathrm{ADHD}$ and the influence of $\mathrm{MPH}$ therapy, which could be important for risk stratification for sudden cardiac death in this patient cohort. In contrast to theoretical speculations that $\mathrm{MPH}$ treatment may increase the risk of sudden death in children with ADHD, our data demonstrate improvement of heart rate variability in Holter ECG. This indicates higher parasympathetic activity induced by $\mathrm{MPH}$, which may be beneficial with respect to the risk of sudden cardiac death in ADHD children and adolescents.

\section{Abbreviations}

ADHD: Attention deficit disorder with or without hyperactivity

ECG: Electrocardiogram

HRV: Heart rate variability

MPH: Methylphenidate

NN: $\quad$ Normal RR intervals

pNN50: Number of pairs of adjacent NN intervals differing by more than $50 \mathrm{~ms}$ divided by the total number of all NN intervals

SDNN: Standard deviation of all NN intervals

rMSSD: Square root of the mean of the sum of the squares of differences between adjacent NN intervals.

\section{Acknowledgments}

The authors wish to thank Dr. Clare Mitchell (Departments of Pediatrics and Psychiatry, The University of Western Ontario, London, Canada) for her critical reviewing of this paper.

\section{References}

[1] S. E. Nissen, "ADHD drugs and cardiovascular risk," New England Journal of Medicine, vol. 354, no. 14, pp. 1445-1448, 2006.

[2] J. K. Triedman and M. E. Alexander, "Needle in a haystack: modeling the incidence of sudden cardiac arrest in healthy children," Circulation, vol. 121, no. 11, pp. 1283-1285, 2010.

[3] M. S. Gould, B. T. Walsh, J. L. Munfakh et al., "Sudden death and use of stimulant medications in youths," American Journal of Psychiatry, vol. 166, no. 9, pp. 992-1001, 2009.

[4] J. Elia and V. L. Vetter, "Cardiovascular effects of medications for the treatment of attention-deficit hyperactivity disorder: what is known and how should it influence prescribing in children?" Pediatric Drugs, vol. 12, no. 3, pp. 165-175, 2010.
[5] P. Denchev, J. R. Kaltman, M. Schoenbaum, and B. Vitiello, "Modeled economic evaluation of alternative strategies to reduce sudden cardiac death among children treated for attention deficit/hyperactivity disorder," Circulation, vol. 121, no. 11, pp. 1329-1337, 2010.

[6] G. Stiefel and F. M. C. Besag, "Cardiovascular effects of methylphenidate, amphetamines and atomoxetine in the treatment of attention-deficit hyperactivity disorder," Drug Safety, vol. 33, no. 10, pp. 821-842, 2010.

[7] J. A. Samuels, K. Franco, F. Wan, and J. M. Sorof, "Effect of stimulants on 24-h ambulatory blood pressure in children with ADHD: a double-blind, randomized, cross-over trial," Pediatric Nephrology, vol. 21, no. 1, pp. 92-95, 2006.

[8] S. Prasad, A. J. Furr, S. Zhang, S. Ball, and A. J. Allen, "Baseline values from the electrocardiograms of children and adolescents with ADHD," Child and Adolescent Psychiatry and Mental Health, vol. 1, article 11, 2007.

[9] L. K. Leslie, M. E. Alexander, T. A. Trikalinos, J. T. Cohen, S. K. Parsons, and J. W. Newburger, "Reexamining the emperor's new clothes: ambiguities in current cardiac screening recommendations for youth with attention deficit hyperactivity disorder," Circulation. Cardiovascular quality and outcomes, vol. 1, no. 2, pp. 134-137, 2008.

[10] M. Vaseghi and K. Shivkumar, "The role of the autonomic nervous system in sudden cardiac death," Progress in Cardiovascular Diseases, vol. 50, no. 6, pp. 404-419, 2008.

[11] M. T. La Rovere, J. T. Bigger Jr., F. I. Marcus, A. Mortara, and P. J. Schwartz, "Baroreflex sensitivity and heart-rate variability in prediction of total cardiac mortality after myocardial infarction," Lancet, vol. 351, no. 9101, pp. 478-484, 1998.

[12] L. Fei, M. H. Anderson, D. Katritsis et al., "Decreased heart rate variability in survivors of sudden cardiac death not associated with coronary artery disease," British Heart Journal, vol. 71, no. 1, pp. 16-21, 1994.

[13] M. Massin and G. Von Bernuth, "Normal ranges of heart rate variability during infancy and childhood," Pediatric Cardiology, vol. 18, no. 4, pp. 297-302, 1997.

[14] K. Umetani, D. H. Singer, R. McCraty, and M. Atkinson, "Twenty-four hour time domain heart rate variability and heart rate: relations to age and gender over nine decades," Journal of the American College of Cardiology, vol. 31, no. 3, pp. 593-601, 1998.

[15] M. Malik, "Heart rate variability: standards of measurement, physiological interpretation, and clinical use," Circulation, vol. 93, no. 5, pp. 1043-1065, 1996.

[16] W. O. Cooper, L. A. Habel, C. M. Sox et al., "ADHD drugs and serious cardiovascular events in children and young adults," New England Journal of Medicine, vol. 365, no. 20, pp. 18961904, 2011.

[17] L. A. Habel, W. O. Cooper, C. M. Sox et al., "ADHD medications and risk of serious cardiovascular events in young and middle-aged adults," Journal of the American Medical Association, vol. 306, no. 24, pp. 2673-2683, 2011.

[18] P. Shaw, "ADHD medications and cardiovascular risk-some heartening news," Journal of the American Medical Association, vol. 306, no. 24, pp. 2723-2724. 

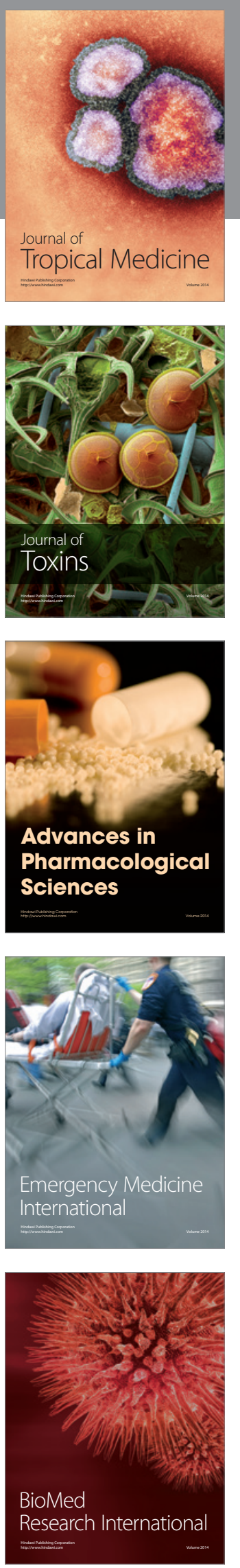
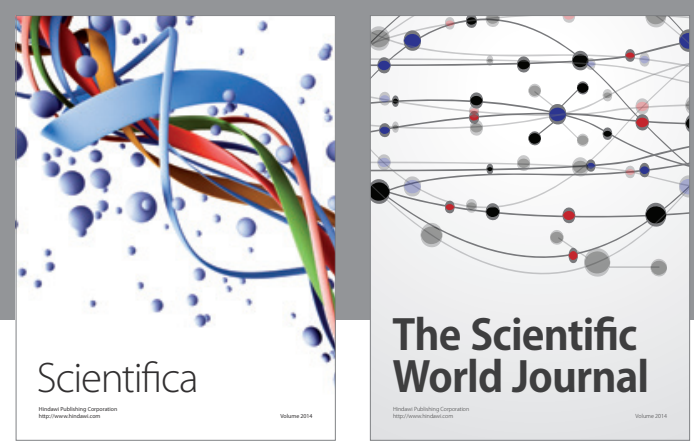

The Scientific World Journal
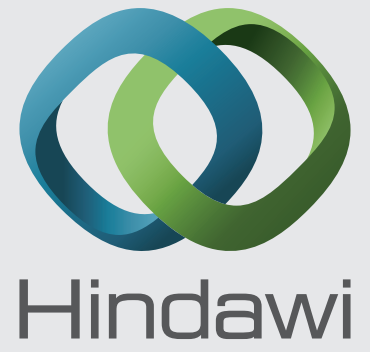

Submit your manuscripts at

http://www.hindawi.com
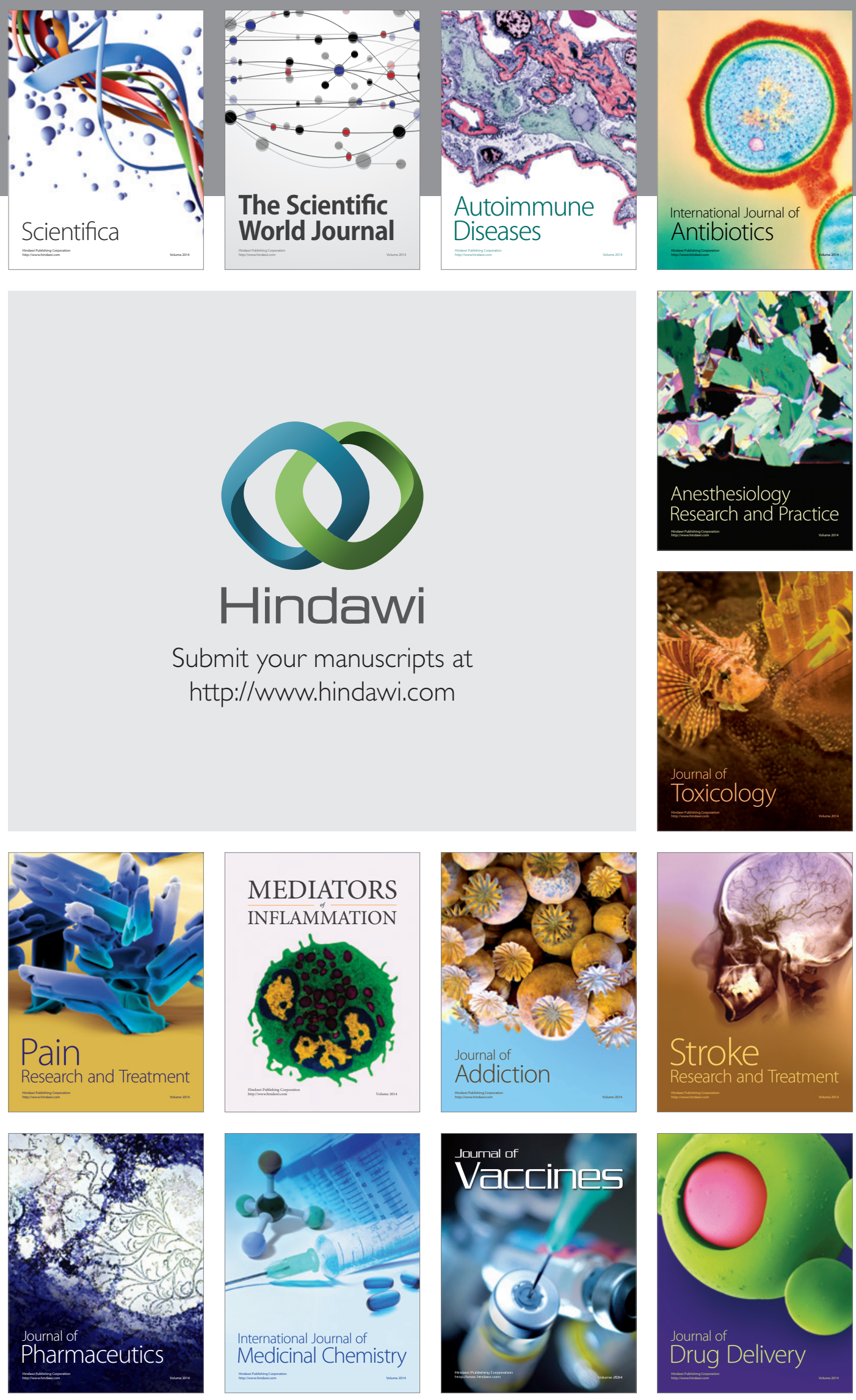